1997-02-01

\title{
Teaching electromagnetic field theory using differential forms
}

\author{
Karl F. Warnick \\ warnick@byu.edu \\ Richard H. Selfridge \\ selfridge@ee.byu.edu \\ David V. Arnold
}

Follow this and additional works at: https://scholarsarchive.byu.edu/facpub

Part of the Electrical and Computer Engineering Commons

\section{Original Publication Citation}

Warnick, K. F., R. H. Selfridge, and D. V. Arnold. "Teaching Electromagnetic Field Theory using Differential Forms." Education, IEEE Transactions on 4.1 (1997): 53-68

\section{BYU ScholarsArchive Citation}

Warnick, Karl F.; Selfridge, Richard H.; and Arnold, David V., "Teaching electromagnetic field theory using differential forms" (1997). Faculty Publications. 669.

https://scholarsarchive.byu.edu/facpub/669 


\title{
Teaching Electromagnetic Field Theory Using Differential Forms
}

\author{
Karl F. Warnick, Richard H. Selfridge, Member, IEEE, and David V. Arnold
}

\begin{abstract}
The calculus of differential forms has significant advantages over traditional methods as a tool for teaching electromagnetic (EM) field theory: First, forms clarify the relationship between field intensity and flux density, by providing distinct mathematical and graphical representations for the two types of fields. Second, Ampere's and Faraday's laws obtain graphical representations that are as intuitive as the representation of Gauss's law. Third, the vector Stokes theorem and the divergence theorem become special cases of a single relationship that is easier for the student to remember, apply, and visualize than their vector formulations. Fourth, computational simplifications result from the use of forms: derivatives are easier to employ in curvilinear coordinates, integration becomes more straightforward, and families of vector identities are replaced by algebraic rules. In this paper, EM theory and the calculus of differential forms are developed in parallel, from an elementary, conceptually oriented point of view using simple examples and intuitive motivations. We conclude that because of the power of the calculus of differential forms in conveying the fundamental concepts of EM theory, it provides an attractive and viable alternative to the use of vector analysis in teaching electromagnetic field theory.
\end{abstract}

\section{INTRODUCTION}

C ERTAIN questions are often asked by students of electromagnetic (EM) field theory: Why does one need both field intensity and flux density to describe a single field? How does one visualize the curl operation? Is there some way to make Ampere's law or Faraday's law as physically intuitive as Gauss's law? The Stokes theorem and the divergence theorem seem vaguely similar; do they have a deeper connection? Because of difficulty with concepts related to these questions, some students leave introductory courses lacking a real understanding of the physics of electromagnetics. Interestingly, none of these concepts are intrinsically more difficult than other aspects of EM theory; rather, they are unclear because of the limitations of the mathematical language traditionally used to teach electromagnetics: vector analysis. In this paper, we show that the calculus of differential forms clarifies these and other fundamental principles of electromagnetic field theory.

The use of the calculus of differential forms in electromagnetics has been explored in several important papers and texts, including Misner, Thorne, and Wheeler [1], Deschamps [2], and Burke [3]. These works note some of the advantages of the use of differential forms in EM theory. Misner et al. and Burke treat the graphical representation of forms and operations on forms, as well as other aspects of the application

Manuscript received June 15, 1994; revised June 19, 1996.

The authors are with the Department of Electrical and Computer Engineering, Brigham Young University, Provo, UT 84602 USA.

Publisher Item Identifier S 0018-9359(97)01545-8. of forms to electromagnetics. Deschamps was among the first to advocate the use of forms in teaching engineering electromagnetics.

Existing treatments of differential forms in EM theory either target an advanced audience or are not intended to provide a complete exposition of the pedagogical advantages of differential forms. This paper presents the topic on an undergraduate level and emphasizes the benefits of differential forms in teaching introductory electromagnetics, especially graphical representations of forms and operators. The calculus of differential forms and principles of EM theory are introduced in parallel, much as would be done in a beginning EM course. We present concrete visual pictures of the various field quantities, Maxwell's laws, and boundary conditions. The aim of this paper is to demonstrate that differential forms are an attractive and viable alternative to vector analysis as a tool for teaching electromagnetic field theory.

\section{A. Development of Differential Forms}

Cartan and others developed the calculus of differential forms in the early 1900's. A differential form is a quantity that can be integrated, including differentials. More precisely, a differential form is a fully covariant, fully antisymmetric tensor. The calculus of differential forms is a self-contained subset of tensor analysis.

Since Cartan's time, the use of forms has spread to many fields of pure and applied mathematics, from differential topology to the theory of differential equations. Differential forms are used by physicists in general relativity [1], quantum field theory [4], thermodynamics [5], mechanics [6], as well as electromagnetics. A section on differential forms is commonplace in mathematical physics texts [7], [8]. Differential forms have been applied to control theory by Hermann [9] and others.

\section{B. Differential Forms in EM Theory}

The laws of electromagnetic field theory as expressed by James Clerk Maxwell in the mid 1800's required dozens of equations. Vector analysis offered a more convenient tool for working with EM theory than earlier methods. Tensor analysis is in turn more concise and general, but is too abstract to give students a conceptual understanding of EM theory. Weyl and Poincaé expressed Maxwell's laws using differential forms early this century. Applied to electromagnetics, differential 
forms combine much of the generality of tensors with the simplicity and concreteness of vectors.

General treatments of differential forms and EM theory include papers [2], [10]-[14]. Ingarden and Jamiołkowski [15] is an electrodynamics text using a mix of vectors and differential forms. Parrott [16] employs differential forms to treat advanced electrodynamics. Thirring [17] is a classical field theory text that includes certain applied topics such as waveguides. Bamberg and Sternberg [5] develop a range of topics in mathematical physics, including EM theory via a discussion of discrete forms and circuit theory. Burke [3] treats a range of physics topics using forms, shows how to graphically represent forms, and gives a useful discussion of twisted differential forms. The general relativity text by Misner, Thorne, and Wheeler [1] has several chapters on EM theory and differential forms, emphasizing the graphical representation of forms. Flanders [6] treats the calculus of forms and various applications, briefly mentioning electromagnetics.

We note here that many authors, including most of those referenced above, give the spacetime formulation of Maxwell's laws using forms, in which time is included as a differential. We use only the $(3+1)$ representation in this paper, since the spacetime representation is treated in many references and is not as convenient for various elementary and applied topics. Other formalisms for EM theory are available, including bivectors, quaternions, spinors, and higher Clifford algebras. None of these offer the combination of concrete graphical representations, ease of presentation, and close relationship to traditional vector methods that the calculus of differential forms brings to undergraduate-level electromagnetics.

The tools of applied electromagnetics have begun to be reformulated using differential forms. The authors have developed a convenient representation of electromagnetic boundary conditions [18]. Thirring [17] treats several applications of EM theory using forms. Reference [19] treats the dyadic Green function using differential forms. Work is also proceeding on the use of Green forms for anisotropic media [20].

\section{Pedagogical Advantages of Differential Forms}

As a language for teaching electromagnetics, differential forms offer several important advantages over vector analysis. Vector analysis allows only two types of quantities: scalar fields and vector fields (ignoring inversion properties). In a three-dimensional space, differential forms of four different types are available. This allows flux density and field intensity to have distinct mathematical expressions and graphical representations, providing the student with mental pictures that clearly reveal the different properties of each type of quantity. The physical interpretation of a vector field is often implicitly contained in the choice of operator or integral that acts on it. With differential forms, these properties are directly evident in the type of form used to represent the quantity.

The basic derivative operators of vector analysis are the gradient, curl, and divergence. The gradient and divergence lend themselves readily to geometric interpretation, but the curl operation is more difficult to visualize. The gradient, curl, and divergence become special cases of a single operator, the exterior derivative, and the curl obtains a graphical representation that is as clear as that for the divergence. The physical meanings of the curl operation and the integral expressions of Faraday's and Ampere's laws become so intuitive that the usual order of development can be reversed by introducing Faraday's and Ampere's laws to students first and using these to motivate Gauss's laws.

The Stokes theorem and the divergence theorem have an obvious connection in that they relate integrals over a boundary to integrals over the region inside the boundary, but in the language of vector analysis they appear very different. These theorems are special cases of the generalized Stokes theorem for differential forms, which also has a simple graphical interpretation.

Since 1992, we have incorporated short segments on differential forms into our beginning, intermediate, and graduate electromagnetics courses. In the Fall of 1995, we reworked the entire beginning electromagnetics course, changing emphasis from vector analysis to differential forms. Following the first semester in which the new curriculum was used, students completed a detailed written evaluation. Out of 44 responses, four were partially negative; the rest were in favor of the change to differential forms. Certainly, enthusiasm of students involved in something new increased the likelihood of positive responses, but one fact was clear: pictures of differential forms helped students understand the principles of electromagnetics.

\section{Outline}

Section II defines differential forms and the degree of a form. Graphical representations for forms of each degree are given, and the differential forms representing the various quantities of electromagnetics are identified. In Section III we use these differential forms to express Maxwell's laws in integral form and give graphical interpretations for each of the laws. Section IV introduces differential forms in curvilinear coordinate systems. Section V applies Maxwell's laws to find the fields due to sources of basic geometries. In Section VI we define the exterior derivative, give the generalized Stokes theorem, and express Maxwell's laws in point form. Section VII treats boundary conditions using the interior product. Section VIII provides a summary of the main points made in the paper.

\section{DifFERENTIAL FormS AND THE ELECTROMAGNETIC FIELD}

In this section we define differential forms of various degrees and identify them with field intensity, flux density, current density, charge density, and scalar potential.

A differential form is a quantity that can be integrated, including differentials. $3 x d x$ is a differential form, as are $x^{2} y d x d y$ and $f(x, y, z) d y d z+g(x, y, z) d z d x$. The type of integral called for by a differential form determines its degree. The form $3 x d x$ is integrated under a single integral over a path and so is a 1 -form. The form $x^{2} y d x d y$ is integrated by a 
TABLE I

Differential Forms of Each Degree

\begin{tabular}{|llcl|}
\hline Degree & Region of Integration & Example & General Form \\
\hline \hline 0-form & Point & $3 x$ & $f(x, y, z, \ldots)$ \\
1-form & Path & $y^{2} d x+z d y$ & $\alpha_{1} d x+\alpha_{2} d y+\alpha_{3} d z$ \\
2-form & Surface & $e^{y} d y d z+e^{x} g d z d x$ & $\beta_{1} d y d z+\beta_{2} d z d x+\beta_{3} d x d y$ \\
3-form & Volume & $(x+y) d x d y d z$ & $g d x d y d z$ \\
\hline
\end{tabular}

TABLE II

The Differential Forms That Represent Fields and Sources

\begin{tabular}{|lllll|}
\hline Quantity & Form & Degree & Units & Vector/Scalar \\
\hline \hline Electric Field Intensity & $\bar{E}$ & 1-form & V & E \\
Magnetic Field Intensity & $H$ & 1-form & A & H \\
Electric Flux Density & $D$ & 2-form & C & D \\
Magnetic Flux Density & $B$ & 2-form & Wb & B \\
Electric Current Density & $J$ & 2-form & A & J \\
Electric Charge Density & $\rho$ & 3-form & C & $q$ \\
\hline
\end{tabular}

double integral over a surface, so its degree is two. A 3-form is integrated by a triple integral over a volume. 0 -forms are functions, "integrated" by evaluation at a point. Table I gives examples of forms of various degrees. The coefficients of the forms can be functions of position, time, and other variables.

\section{A. Representing the Electromagnetic Field with Differential Forms}

From Maxwell's laws in integral form, we can readily determine the degrees of the differential forms that will represent the various field quantities. In vector notation,

$$
\begin{aligned}
& \oint_{P} \boldsymbol{E} \cdot d \boldsymbol{l}=-\frac{d}{d t} \int_{A} \boldsymbol{B} \cdot d \boldsymbol{A} \\
& \oint_{P} \boldsymbol{H} \cdot d \boldsymbol{l}=\frac{d}{d t} \int_{A} \boldsymbol{D} \cdot d \boldsymbol{A}+\int_{A} \boldsymbol{J} \cdot d \boldsymbol{A} \\
& \oint_{S} \boldsymbol{D} \cdot d \boldsymbol{S}=\int_{V} q d v \\
& \oint_{S} \boldsymbol{B} \cdot d \boldsymbol{S}=0
\end{aligned}
$$

where $A$ is a surface bounded by a path $P, V$ is a volume bounded by a surface $S, q$ is volume charge density, and the other quantities are defined as usual. The electric field intensity is integrated over a path, so that it becomes a 1form. The magnetic field intensity is also integrated over a path, and becomes a 1-form as well. The electric and magnetic flux densities are integrated over surfaces, and so are 2-forms. The sources are electric current density, which is a 2 -form, since it falls under a surface integral, and the volume charge density, which is a 3 -form, as it is integrated over a volume. Table II summarizes these forms.

\section{B. 1-Forms: Field Intensity}

The usual physical motivation for electric field intensity is the force experienced by a small test charge placed in the field. This leads naturally to the vector representation of the electric field, which might be called the "force picture." Another physical viewpoint for the electric field is the change in potential experienced by a charge as it moves through the

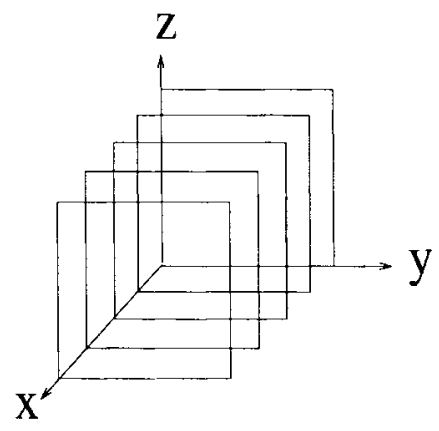

(a)

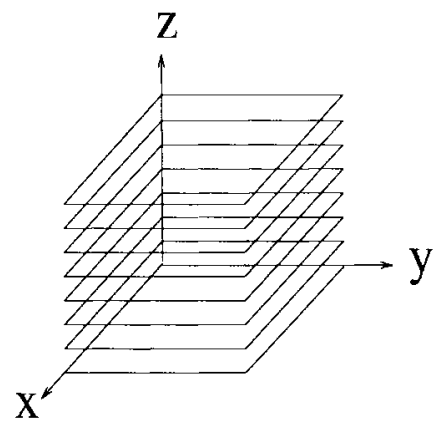

(b)

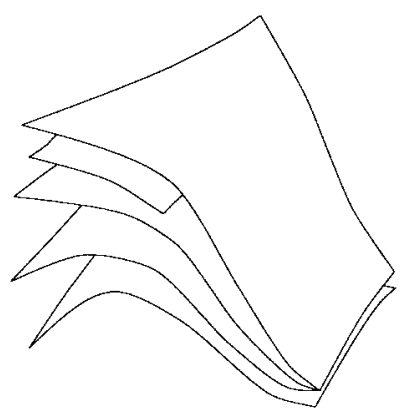

(c)

Fig. 1. (a) The 1-form $d x$, with surfaces perpendicular to the $x$-axis and infinite in the $y$ and $z$ directions. (b) The 1 -form $2 d z$, with surfaces perpendicular to the $z$-axis and spaced two per unit distance in the $z$ direction. (c) A general 1-form, with curved surfaces and surfaces that end or meet each other.

field. This leads naturally to the equipotential representation of the field, or the "energy picture." The energy picture shifts emphasis from the local concept of force experienced by a test charge to the global behavior of the field as manifested by change in energy of a test charge as it moves along a path.

Differential forms lead to the "energy picture" of field intensity. A 1-form is represented graphically as surfaces in space [1], [3]. For a conservative field, the surfaces of the associated 1-form are equipotentials. The differential $d x$ produces surfaces perpendicular to the $x$-axis, as shown in Fig. 1(a). Likewise, $d y$ has surfaces perpendicular to the $y$ axis and the surfaces of $d z$ are perpendicular to the $z$-axis. A linear combination of these differentials has surfaces that are skew to the coordinate axes. The coefficients of a 1form determine the spacing of the surfaces per unit length; the greater the magnitude of the coefficients, the more closely spaced are the surfaces. The 1-form $2 d z$, shown in Fig. 1(b), has surfaces spaced twice as closely as those of $d x$ in Fig. 1(a).

In general, the surfaces of a 1-form can curve, end, or meet each other, depending on the behavior of the coefficients 


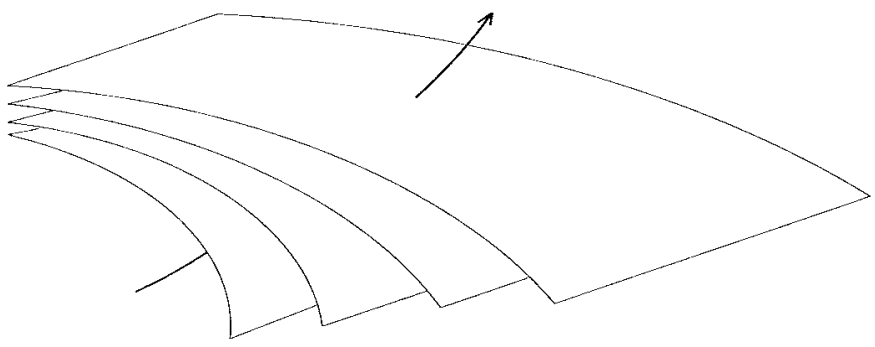

Fig. 2. A path piercing four surfaces of a 1-form. The integral of the 1-form over the path is four.

of the form. If surfaces of a 1-form do not meet or end, the field represented by the form is conservative. The field corresponding to the 1 -form in Fig. 1(a) is conservative; the field in Fig. 1(c) is nonconservative.

Just as a line representing the magnitude of a vector has two possible orientations, the surfaces of a 1-form are oriented as well. This is done by specifying one of the two normal directions to the surfaces of the form. The surfaces of $3 d x$ are oriented in the $+x$ direction, and those of $-3 d x$ in the $-x$ direction. The orientation of a form is usually clear from context and is omitted from figures.

Differential forms are by definition the quantities that can be integrated, so it is natural that the surfaces of a 1-form are a graphical representation of path integration. The integral of a 1-form along a path is the number of surfaces pierced by the path (Fig. 2), taking into account the relative orientations of the surfaces and the path. This simple picture of path integration will provide in the next section a means for visualizing Ampere's and Faraday's laws.

The 1-form $E_{1} d x+E_{2} d y+E_{3} d z$ is said to be dual to the vector field $E_{1} \hat{\boldsymbol{x}}+E_{2} \hat{\boldsymbol{y}}+E_{3} \hat{\boldsymbol{z}}$. The field intensity 1-forms $E$ and $H$ are dual to the vectors $\boldsymbol{E}$ and $\boldsymbol{H}$.

Following Deschamps, we take the units of the electric and magnetic field intensity 1-forms to be volts and amperes, as shown in Table II. The differentials are considered to have units of length. Other field and source quantities are assigned units according to this same convention. A disadvantage of Deschamps' system is that it implies in a sense that the metric of space carries units. Alternative conventions are available; Bamberg and Sternberg [5] and others take the units of the electric and magnetic field intensity 1-forms to be volts per meter and amperes per meter, the same as their vector counterparts, so that the differentials carry no units and the integration process itself is considered to provide a factor of length. If this convention is chosen, the basis differentials of curvilinear coordinate systems (see Section IV) must also be taken to carry no units. This leads to confusion for students, since these basis differentials can include factors of distance. The advantages of this alternative convention are that it is more consistent with the mathematical point of view, in which basis vectors and forms are abstract objects not associated with a particular system of units, and that a field quantity has the same units whether represented by a vector or a differential form. Furthermore, a general differential form may include differentials of functions that do not represent

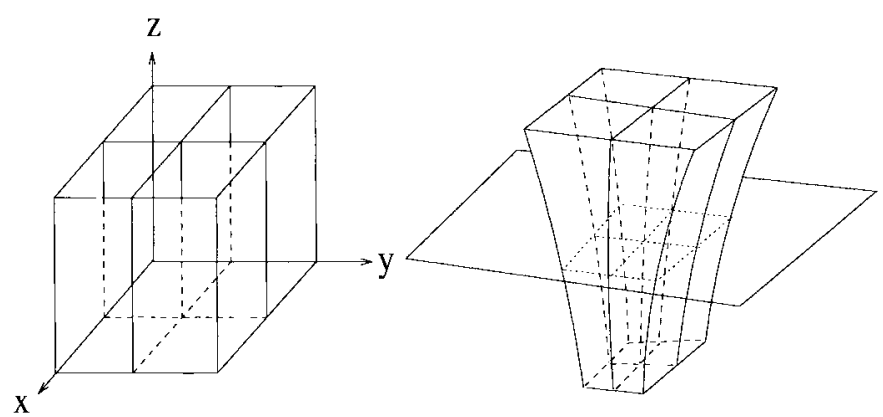

(a)

(b)

Fig. 3. (a) The 2 -form $d x d y$, with tubes in the $z$ direction. (b) Four tubes of a 2 -form pass through a surface, so that the integral of the 2 -form over the surface is four.

position and so cannot be assigned units of length. The possibility of confusion when using curvilinear coordinates seems to outweigh these considerations, and so we have chosen Deschamps' convention.

With this convention, the electric field intensity 1-form can be taken to have units of energy per charge, or joules per coulomb. This supports the "energy picture," in which the electric field represents the change in energy experienced by a charge as it moves through the field. One might argue that this motivation of field intensity is less intuitive than the concept of force experienced by a test charge at a point. While this may be true, the graphical representations of Ampere's and Faraday's laws that will be outlined in Section III favor the differential form point of view. Furthermore, the simple correspondence between vectors and forms allows both to be introduced with little additional effort, providing students a more solid understanding of field intensity than they could obtain from one representation alone.

\section{2-Forms: Flux Density and Current Density}

Flux density or flow of current can be thought of as tubes that connect sources of flux or current. This is the natural graphical representation of a 2-form, which is drawn as sets of surfaces that intersect to form tubes. The differential $d x d y$ is represented by the surfaces of $d x$ and $d y$ superimposed. The surfaces of $d x$ perpendicular to the $x$-axis and those of $d y$ perpendicular to the $y$-axis intersect to produce tubes in the $z$ direction, as illustrated by Fig. 3(a). (To be precise, the tubes of a 2-form have no definite shape: tubes of $d x d y$ have the same density those of $[0.5 d x][2 d y]$.) The coefficients of a 2form give the spacing of the tubes. The greater the coefficients, the more dense the tubes. An arbitrary 2-form has tubes that may curve or converge at a point.

The direction of flow or flux along the tubes of a 2-form is given by the right-hand rule applied to the orientations of the surfaces making up the walls of a tube. The orientation of $d x$ is in the $+x$ direction, and $d y$ in the $+y$ direction, so the flux due to $d x d y$ is in the $+z$ direction.

As with 1-forms, the graphical representation of a 2-form is fundamentally related to the integration process. The integral of a 2 -form over a surface is the number of tubes passing through the surface, where each tube is weighted positively if 


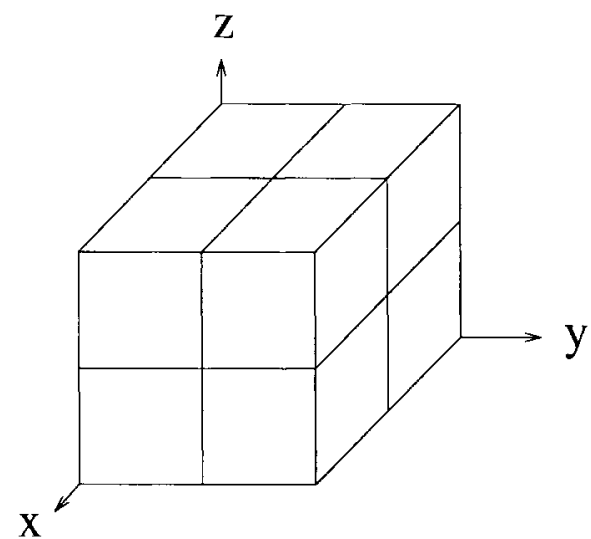

Fig. 4. The 3-form $d x d y d z$, with cubes of side equal to one. The cubes fill all space.

its orientation is in the direction of the surface's oriention, and negatively if opposite. This is illustrated in Fig. 3(b).

As with 1-forms, 2-forms correspond to vector fields in a simple way. An arbitrary 2-form $D_{1} d y d z+D_{2} d z d x+$ $D_{3} d x d y$ is dual to the vector field $D_{1} \hat{\boldsymbol{x}}+D_{2} \hat{\boldsymbol{y}}+D_{3} \hat{z}$, so that the flux density 2 -forms $D$ and $B$ are dual to the usual flux density vectors $\boldsymbol{D}$ and $\boldsymbol{B}$.

\section{3-Forms: Charge Density}

Some scalar physical quantities are densities, and can be integrated over a volume. For other scalar quantities, such as electric potential, a volume integral makes no sense. The calculus of forms distinguishes between these two types of quantities by representing densities as 3 -forms. Volume charge density, for example, becomes

$$
\rho=q d x d y d z
$$

where $q$ is the usual scalar charge density in the notation of [2].

A 3-form is represented by three sets of surfaces in space that intersect to form boxes. The density of the boxes is proportional to the coefficient of the 3-form; the greater the coefficient, the smaller and more closely spaced are the boxes. A point charge is represented by an infinitesimal box at the location of the charge. The 3 -form $d x d y d z$ is the union of three families of planes perpendicular to each of the $x, y$, and $z$ axes. The planes along each of the axes are spaced one unit apart, forming cubes of unit side distributed evenly throughout space, as in Fig. 4. The orientation of a 3-form is given by specifying the sign of its boxes. As with other differential forms, the orientation is usually clear from context and is omitted from figures.

\section{E. 0-Forms: Scalar Potential}

0 -forms are functions. The scalar potential $\phi$, for example, is a 0 -form. Any scalar physical quantity that is not a volume density is represented by a 0 -form.

\section{F. Summary}

The use of differential forms helps students to understand electromagnetics by giving them distinct mental pictures that they can associate with the various fields and sources. As vectors, field intensity and flux density are mathematically and graphically indistinguishable as far as the type of physical quantity they represent. As differential forms, the two types of quantities have graphical representations that clearly express the physical meaning of the field. The surfaces of a field intensity 1-form assign potential change to a path. The tubes of a flux density 2-form give the total flux or flow through a surface. Charge density is also distinguished from other types of scalar quantities by its representation as a 3 -form.

\section{MAXWELL'S LAWS IN INTEGRAL FORM}

In this section, we discuss Maxwell's laws in integral form in light of the graphical representations given in the previous section. Using the differential forms defined in Table II, Maxwell's laws can be written

$$
\begin{aligned}
& \oint_{P} E=-\frac{d}{d t} \int_{A} B \\
& \oint_{P} H=\frac{d}{d t} \int_{A} D+\int_{A} J \\
& \oint_{S} D=\int_{V} \rho \\
& \oint_{S} B=0 .
\end{aligned}
$$

The first pair of laws is often more difficult for students to grasp than the second, because the vector picture of curl is not as intuitive as that for divergence. With differential forms, Ampere's and Faraday's laws are graphically very similar to Gauss's laws for the electric and magnetic fields. The close relationship between the two sets of laws becomes clearer.

\section{A. Ampere's and Faraday's Laws}

Faraday's and Ampere's laws equate the number of surfaces of a 1-form pierced by a closed path to the number of tubes of a 2 -form passing through the path. Each tube of $J$, for example, must have a surface of $H$ extending away from it, so that any path around the tube pierces the surface of $H$. Thus Ampere's law states that tubes of displacement current and electric current are sources for surfaces of $H$. This is illustrated in Fig. 5(a). Likewise, tubes of time-varying magnetic flux density are sources for surfaces of $E$.

The illustration of Ampere's law in Fig. 5(a) is arguably the most important pedagogical advantage of the calculus of differential forms over vector analysis. Ampere's and Faraday's laws are usually considered the more difficult pair of Maxwell's laws, because vector analysis provides no simple picture that makes the physical meaning of these laws intuitive. Compare Fig. 5(a) to the vector representation of the same field in Fig. 5(b). The vector field appears to "curl" everywhere in space. Students must be convinced that indeed the field has no curl except at the location of the current, using some pedagogical device such as an imaginary paddle wheel in a rotating fluid. The surfaces of $H$, on the other hand, end only along the tubes of current; where they do not end, the field has no curl. This is the fundamental concept underlying 


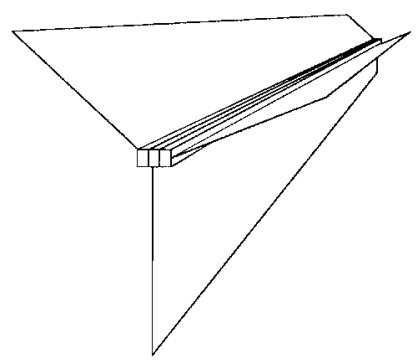

(a)

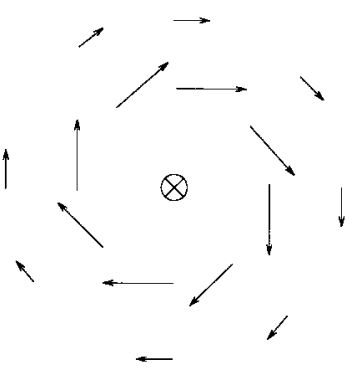

(b)
Fig. 5. (a) A graphical representation of Ampere's law: tubes of current produce surfaces of magnetic field intensity. Any loop around the three tubes of $J$ must pierce three surfaces of $H$. (b) A cross section of the same magnetic field using vectors. The vector field appears to "curl" everywhere, even though the field has nonzero curl only at the location of the current.

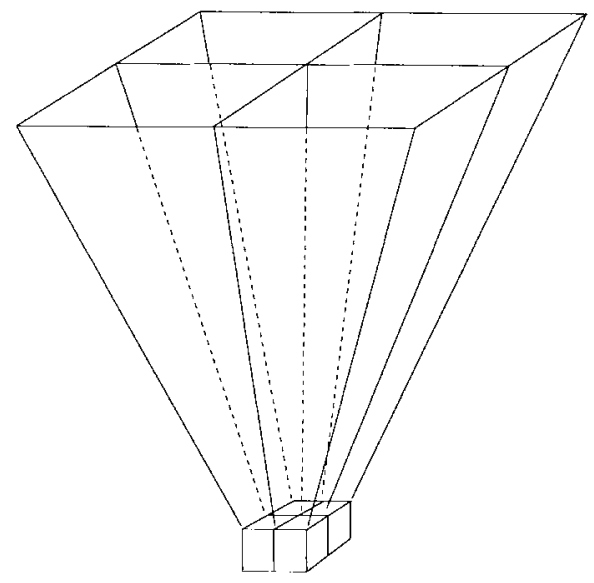

Fig. 6. A graphical representation of Gauss's law for the electric flux density: boxes of $\rho$ produce tubes of $D$.

Ampere's and Faraday's laws: tubes of time-varying flux or current produce field intensity surfaces.

\section{B. Gauss's Laws}

Gauss's law for the electric field states that the number of tubes of $D$ flowing out through a closed surface must be equal to the number of boxes of $\rho$ inside the surface. The boxes of $\rho$ are sources for the tubes of $D$, as shown in Fig. 6. Gauss's law for the magnetic flux density states that tubes of the 2form $B$ can never end - they must either form closed loops or go off to infinity.

Comparing Figs. 5(a) and 6 shows the close relationship between the two sets of Maxwell's laws. In the same way that flux density tubes are produced by boxes of electric charge, field intensity surfaces are produced by tubes of the sources on the right-hand sides of Faraday's and Ampere's laws. Conceptually, the laws only differ in the degrees of the forms involved and the dimensions of their pictures.

\section{Constitutive Relations and the Star Operator}

The usual vector expressions of the constitutive relations for an isotropic medium,

$$
\begin{aligned}
& \boldsymbol{D}=\epsilon \boldsymbol{E} \\
& \boldsymbol{B}=\mu \boldsymbol{H}
\end{aligned}
$$

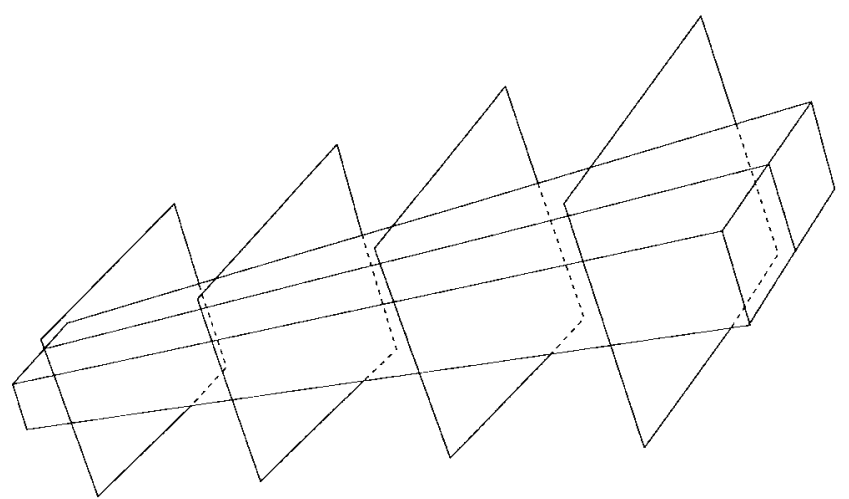

Fig. 7. The star operator relates 1 -form surfaces to perpendicular 2 -form tubes.

involve scalar multiplication. With differential forms, we cannot use these same relationships, because $D$ and $B$ are 2-forms, while $E$ and $H$ are 1-forms. An operator that relates forms of different degrees must be introduced.

The Hodge star operator [5], [17] naturally fills this role. As vector spaces, the spaces of 0 -forms and 3-forms are both onedimensional, and the spaces of 1-forms and 2-forms are both three-dimensional. The star operator $\star$ is a set of isomorphisms between these pairs of vector spaces.

For 1-forms and 2-forms, the star operator satisfies

$$
\begin{aligned}
\star d x & =d y d z \\
\star d y & =d z d x \\
\star d z & =d x d y .
\end{aligned}
$$

0 -forms and 3 -forms are related by

$$
\star 1=d x d y d z .
$$

In $R^{3}$, the star operator is its own inverse, so that $\star \star \alpha=\alpha$. A 1 -form $\omega$ is dual to the same vector as the 2 -form $\star \omega$.

Graphically, the star operator replaces the surfaces of a form with orthogonal surfaces, as in Fig. 7. The 1-form $3 d x$, for example, has planes perpendicular to the $x$-axis. It becomes $3 d y d z$ under the star operation. This 2 -form has planes perpendicular to the $y$ and the $z$ axes.

Using the star operator, the constitutive relations are

$$
\begin{aligned}
& D=\epsilon \star E \\
& B=\mu \star H
\end{aligned}
$$

where $\epsilon$ and $\mu$ are the permittivity and permeability of the medium. The surfaces of $E$ are perpendicular to the tubes of $D$, and the surfaces of $H$ are perpendicular to the tubes of $B$. The following example illustrates the use of these relations.

Example 1 (Finding D Due to an Electric Field Intensity): Let $E=(d x+d y) e^{i k(x-y)}$ volts be the electric field in free space. We wish to find the flux density due to this field. Using the constitutive relationship between $D$ and $E$

$$
\begin{aligned}
D & =\epsilon_{0} \star(d x+d y) e^{i k(x-y)} \\
& =\epsilon_{0} e^{i k(x-y)}(\star d x+\star d y) \\
& =\epsilon_{0} e^{i k(x-y)}(d y d z+d z d x) \quad \text { (C). }
\end{aligned}
$$


While we restrict our attention to isotropic media in this paper, the star operator applies equally well to anisotropic media. As discussed in [5] and elsewhere, the star operator depends on a metric. If the metric is related to the permittivity or the permeability tensor, anisotropic star operators are obtained, and the constitutive relations become $D=\star_{e} E$ and $B=\star_{h} H$ [20]. Graphically, an anisotropic star operator acts on 1-form surfaces to produce 2 -form tubes that intersect the surfaces obliquely rather than orthogonally.

\section{The Exterior Product and the Poynting 2-form}

Between the differentials of 2 -forms and 3 -forms is an implied exterior product, denoted by a wedge $\wedge$. The wedge is nearly always omitted from the differentials of a form, especially when the form appears under an integral sign. The exterior product of 1 -forms is anticommutative, so that $d x \wedge d y=-d y \wedge d x$. As a consequence, the exterior product is in general supercommutative, so that

$$
\alpha \wedge \beta=(-1)^{a b} \beta \wedge \alpha
$$

where $a$ and $b$ are the degrees of $\alpha$ and $\beta$, respectively. One usually converts the differentials of a form to right-cyclic order using (5).

As a consequence of (5), any differential form with a repeated differential vanishes. In a three-dimensional space each term of a $p$-form will always contain a repeated differential if $p>3$, so there are no nonzero $p$-forms for $p>3$.

The exterior product of two 1-forms is analogous to the vector cross product. With vector analysis, it is not obvious that the cross product of vectors is a different type of quantity than the factors. Under coordinate inversion, $\boldsymbol{a} \times \boldsymbol{b}$ changes sign relative to a vector with the same components, so that $\boldsymbol{a} \times \boldsymbol{b}$ is a pseudovector. With forms, the distinction between $a \wedge b$ and $a$ or $b$ individually is clear.

The exterior product of a 1-form and a 2-form corresponds to the dot product. The coefficient of the resulting 3 -form is equal to the dot product of the vector fields dual to the 1-form and 2 -form in the Euclidean metric.

Combinations of cross and dot products are somewhat difficult to manipulate algebraically, often requiring the use of tabulated identities. Using the supercommutativity of the exterior product, the student can easily manipulate arbitrary products of forms. For example, the identities

$$
A \cdot(B \times C)=C \cdot(A \times B)=B \cdot(C \times A)
$$

are special cases of

$$
A \wedge B \wedge C=C \wedge A \wedge B=B \wedge C \wedge A
$$

where $A, B$, and $C$ are forms of arbitrary degrees. The factors can be interchanged easily using (5).

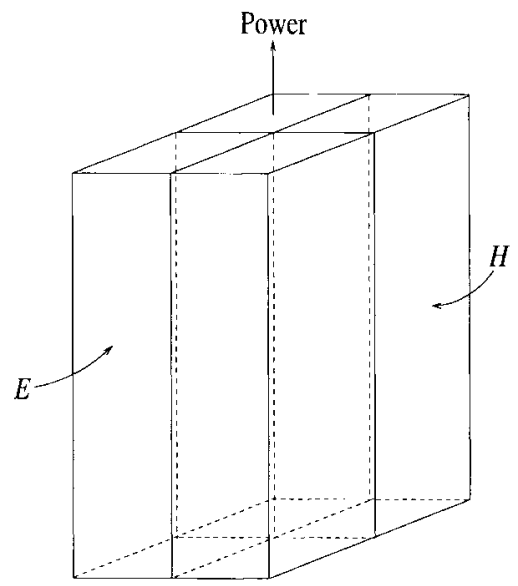

Fig. 8. The Poynting power flow 2 -form $S=E \wedge H$. Surfaces of the 1 -forms $E$ and $H$ are the sides of the tubes of $S$.

Consider the exterior product of the 1-forms $E$ and $H$

$$
\begin{aligned}
E \wedge H= & \left(E_{1} d x+E_{2} d y+E_{3} d z\right) \\
& \wedge\left(H_{1} d x+H_{2} d y+H_{3} d z\right) \\
= & E_{1} H_{1} d x d x+E_{1} H_{2} d x d y+E_{1} H_{3} d x d z \\
& +E_{2} H_{1} d y d x+E_{2} H_{2} d y d y+E_{2} H_{3} d y d z \\
& +E_{3} H_{1} d z d x+E_{3} H_{2} d z d y+E_{3} H_{3} d z d z \\
= & \left(E_{2} H_{3}-E_{3} H_{2}\right) d y d z+\left(E_{3} H_{1}-E_{1} H_{3}\right) d z d x \\
& +\left(E_{1} H_{2}-E_{2} H_{1}\right) d x d y .
\end{aligned}
$$

This is the Poynting 2-form $S$. For complex fields, $S=$ $E \wedge H^{*}$. For time-varying fields, the tubes of this 2 -form represent flow of electromagnetic power, as shown in Fig. 8. The sides of the tubes are the surfaces of $E$ and $H$. This gives a clear geometrical interpretation to the fact that the direction of power flow is orthogonal to the orientations of both $E$ and $H$.

Example 2 (The Poynting 2-Form Due to a Plane Wave):

Consider a plane wave propagating in free space in the $z$ direction, with the time-harmonic electric field $E=E_{0} d x$ volts in the $x$ direction. The Poynting 2 -form is

$$
\begin{aligned}
S & =E \wedge H \\
& =E_{0} d x \wedge \frac{E_{0}}{\eta_{0}} d y \\
& =\frac{E_{0}^{2}}{\eta_{0}} d x d y \quad(\mathrm{~W})
\end{aligned}
$$

where $\eta_{0}$ is the wave impedance of free space.

\section{E. Energy Density}

The exterior products $E \wedge D$ and $H \wedge B$ are 3-forms that represent the density of electromagnetic energy. The energy density 3 -form $w$ is defined to be

$$
w=\frac{1}{2}(E \wedge D+H \wedge B) .
$$

The volume integral of $w$ gives the total energy stored in a region of space by the fields present in the region.

Fig. 9 shows the energy density 3 -form between the plates of a capacitor, where the upper and lower plates are equally 


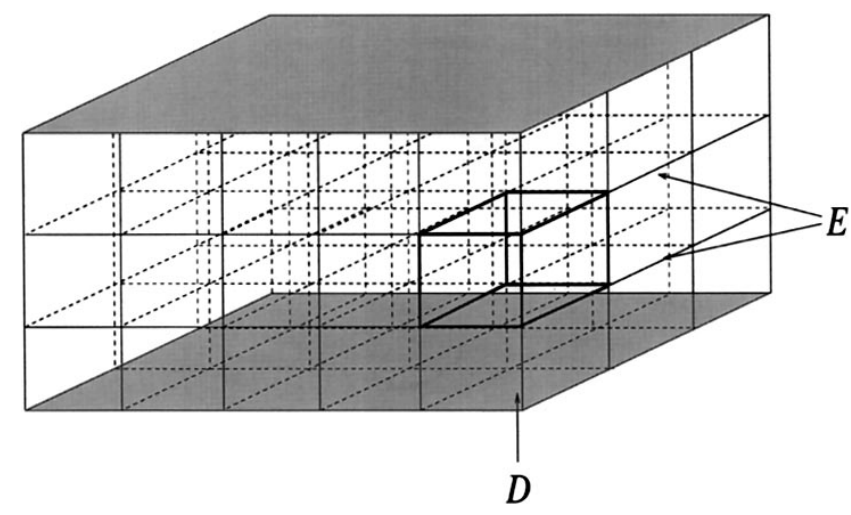

Fig. 9. The 3-form $2 w$ due to fields inside a parallel-plate capacitor with oppositely charged plates. The surfaces of $E$ are parallel to the top and bottom plates. The tubes of $D$ extend vertically from charges on one plate to opposite charges on the other. The tubes and surfaces intersect to form cubes of $2 \omega$, one of which is outlined in the figure.

and oppositely charged. The boxes of $2 w$ are the intersection of the surfaces of $E$, which are parallel to the plates, with the tubes of $D$, which extend vertically from one plate to the other.

\section{Curvilinear CoORdinate Systems}

In this section, we give the basis differentials, the star operator, and the correspondence between vectors and forms for cylindrical, spherical, and generalized orthogonal coordinates.

\section{A. Cylindrical Coordinates}

The differentials of the cylindrical coordinate system are $d \rho, \rho d \phi$, and $d z$. Each of the basis differentials is considered to have units of length. The general 1-form

$$
A d \rho+B \rho d \phi+C d z
$$

is dual to the vector

$$
A \hat{\boldsymbol{\rho}}+B \hat{\boldsymbol{\phi}}+C \hat{z} .
$$

The general 2 -form

$$
A \rho d \phi \wedge d z+B d z \wedge d \rho+C d \rho \wedge \rho d \phi
$$

is dual to the same vector. The 2 -form $d \rho d \phi$, for example, is dual to the vector $(1 / \rho) \hat{\boldsymbol{z}}$.

Differentials must be converted to basis elements before the star operator is applied. The star operator in cylindrical coordinates acts as follows:

$$
\begin{aligned}
\star d \rho & =\rho d \phi \wedge d z \\
\star \rho d \phi & =d z \wedge d \rho \\
\star d z & =d \rho \wedge \rho d \phi .
\end{aligned}
$$

Also, $\star 1=\rho d \rho d \phi d z$. As with the rectangular coordinate system, $\star \star=1$. The star operator applied to $d \phi d z$, for example, yields $(1 / \rho) d \rho$.

Fig. 10 shows the pictures of the differentials of the cylindrical coordinate system. The 2 -forms can be obtained by superimposing these surfaces. Tubes of $d z \wedge d \rho$, for example, are square rings formed by the union of Figs. 10(a) and 10(c).

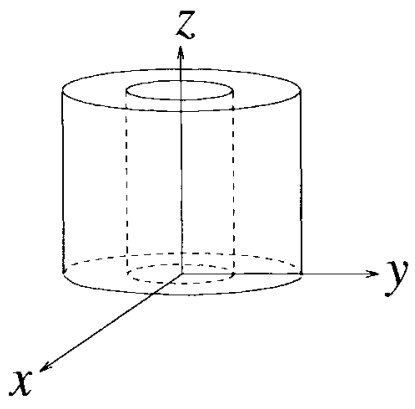

(a)

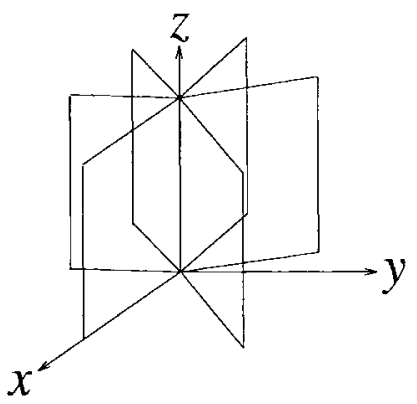

(b)

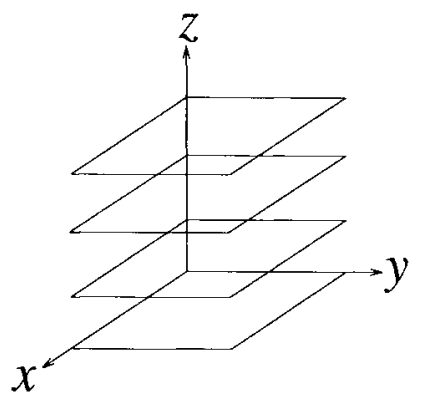

(c)

Fig. 10. Surfaces of (a) $d \rho$, (b) $d \phi$ scaled by $3 / \pi$, and (c) $d z$.

\section{B. Spherical Coordinates}

The basis differentials of the spherical coordinate system are $d r, r d \theta$ and $r \sin \theta d \phi$, each having units of length. The 1 -form

$$
A d r+B r d \theta+C r \sin \theta d \phi
$$

and the 2 -form

$$
A r d \theta \wedge r \sin \theta d \phi+B r \sin \theta d \phi \wedge d r+C d r \wedge r d \theta
$$

are both dual to the vector

$$
A \hat{\boldsymbol{r}}+B \hat{\boldsymbol{\theta}}+C \hat{\boldsymbol{\phi}}
$$

so that $d \theta d \phi$, for example, is dual to the vector $\hat{\boldsymbol{r}} /\left(r^{2} \sin \theta\right)$.

As in the cylindrical coordinate system, differentials must be converted to basis elements before the star operator is applied. The star operator acts on 1-forms and 2-forms as follows:

$$
\begin{aligned}
\star d r & =r d \theta \wedge r \sin \theta d \phi \\
\star r d \theta & =r \sin \theta d \phi \wedge d r \\
\star r \sin \theta d \phi & =d r \wedge r d \theta .
\end{aligned}
$$

Again, $\star \star=1$. The star operator applied to one is

$$
\star 1=r^{2} \sin \theta d r d \theta d \phi
$$

Fig. 11 shows the pictures of the differentials of the spherical coordinate system; pictures of 2 -forms can be obtained by superimposing these surfaces. 


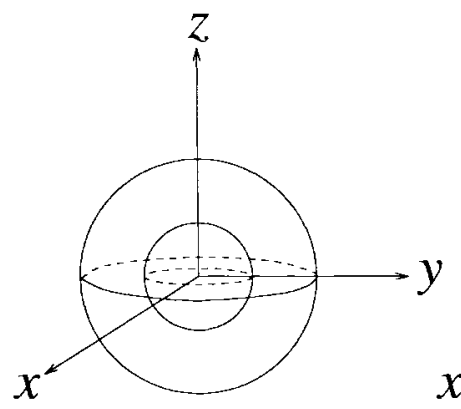

(a)

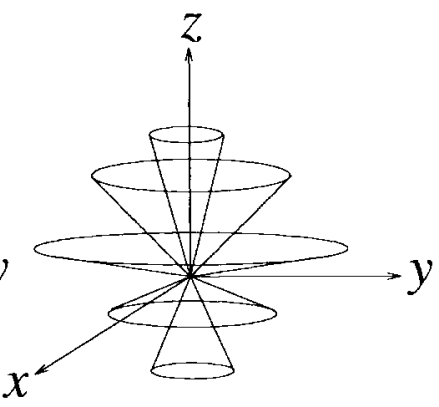

(b)

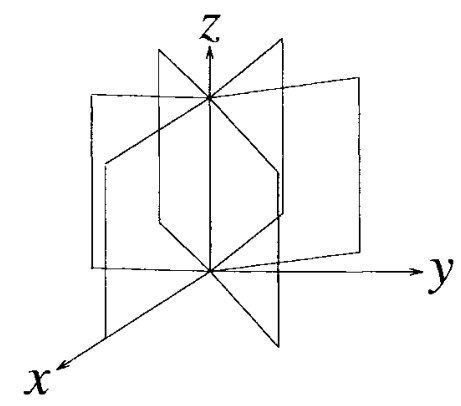

(c)

Fig. 11. Surfaces of (a) $d r$, (b) $d \theta$ scaled by $10 / \pi$, and (c) $d \phi$ scaled by $3 / \pi$.

\section{Generalized Orthogonal Coordinates}

Let the location of a point be given by $(u, v, w)$ such that the tangents to each of the coordinates are mutually orthogonal. Define a function $h_{1}$ such that the integral of $h_{1} d u$ along any path with $v$ and $w$ constant gives the length of the path. Define $h_{2}$ and $h_{3}$ similarly. Then the basis differentials are

$$
h_{1} d u, h_{2} d v, h_{3} d w .
$$

The 1-form $A h_{1} d u+B h_{2} d v+C h_{3} d w$ and the 2-form $A h_{2} h_{3} d v \wedge d w+B h_{3} h_{1} d w \wedge d u+C h_{1} h_{2} d u \wedge d v$ are both dual to the vector $A \hat{\boldsymbol{u}}+B \hat{\boldsymbol{v}}+C \hat{\boldsymbol{w}}$. The star operator on 1-forms and 2 -forms satisfies

$$
\begin{aligned}
& \star\left(A h_{1} d u+B h_{2} d v+C h_{3} d w\right) \\
& \quad=A h_{2} h_{3} d v \wedge d w+B h_{3} h_{1} d w \wedge d u+C h_{1} h_{2} d u \wedge d v .
\end{aligned}
$$

For 0-forms and 3-forms, $\star 1=h_{1} h_{2} h_{3} d u d v d w$.

\section{Electrostatics and Magnetostatics}

In this section we treat several of the usual elementary applications of Maxwell's laws in integral form. We find the electric flux due to a point charge and a line charge using Gauss's law for the electric field. Ampere's law is used to find the magnetic fields produced by a line current.

\section{A. Point Charge}

By symmetry, the tubes of flux from a point charge $Q$ must extend out radially from the charge (Fig. 12), so that

$$
D=D_{0} r^{2} \sin \theta d \theta d \phi
$$

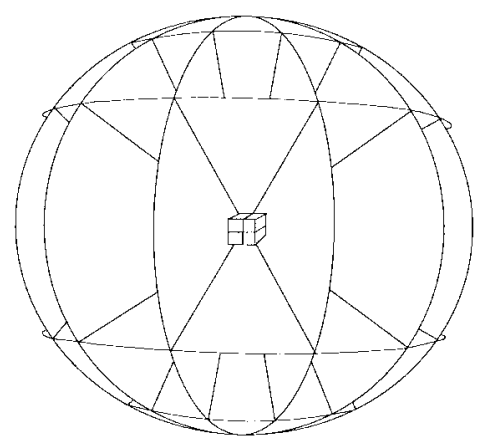

Fig. 12. Electric flux density due to a point charge. Tubes of $D$ extend away from the charge.

To apply Gauss's law

$$
\oint_{S} D=\int_{V} \rho
$$

we choose $S$ to be a sphere enclosing the charge. The righthand side of Gauss's law is equal to $Q$, and the left-hand side is

$$
\begin{aligned}
\oint_{S} D & =\int_{0}^{2 \pi} \int_{0}^{\pi} D_{0} r^{2} \sin \theta d \theta d \phi \\
& =4 \pi r^{2} D_{0} .
\end{aligned}
$$

Solving for $D_{0}$ and substituting into (15)

$$
D=\frac{Q}{4 \pi r^{2}} r d \theta r \sin \theta d \phi(\mathrm{C})
$$

for the electric flux density due to the point charge. This can also be written

$$
D=\frac{Q}{4 \pi} \sin \theta d \theta d \phi(\mathrm{C}) .
$$

Since $4 \pi$ is the total amount of solid angle for a sphere and $\sin \theta d \theta d \phi$ is the differential element of solid angle, this expression matches Fig. 12 in showing that the amount of flux per solid angle is constant.

\section{B. Line Charge}

For a line charge with charge density $\rho_{l}$ coulombs per meter, by symmetry tubes of flux extend out radially from the line, as shown in Fig. 13. The tubes are bounded by the surfaces of $d \phi$ and $d z$, so that $D$ has the form

$$
D=D_{0} d \phi d z .
$$

Let $S$ be a cylinder of height $b$ with the line charge along its axis. The right-hand side of Gauss's law is

$$
\begin{aligned}
\int_{V} \rho & =\int_{0}^{b} \rho_{l} d z \\
& =b \rho_{l} .
\end{aligned}
$$

The left-hand side is

$$
\begin{aligned}
\oint_{S} D & =\int_{0}^{b} \int_{0}^{2 \pi} D_{0} d \phi d z \\
& =2 \pi b D_{0} .
\end{aligned}
$$




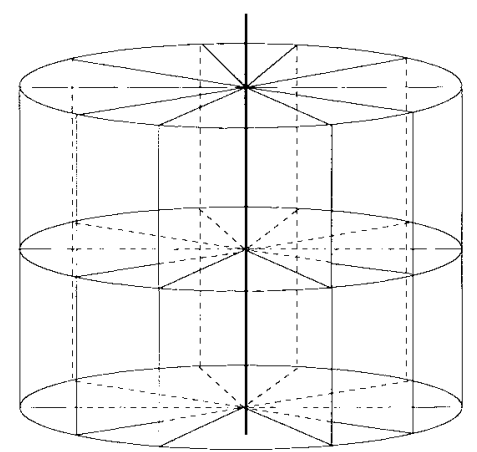

Fig. 13. Electric flux density due to a line charge. Tubes of $D$ extend radially away from the vertical line of charge.

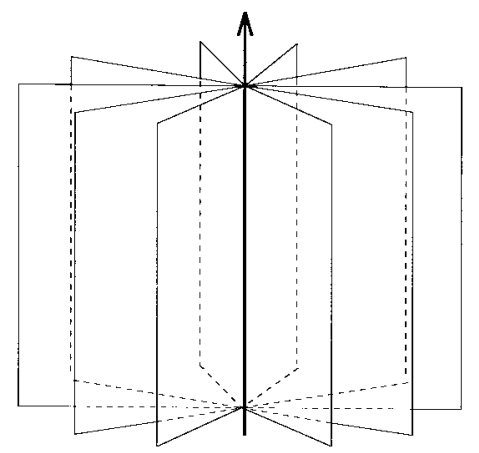

Fig. 14. Magnetic field intensity $H$ due to a line current.

Solving for $D_{0}$ and substituting into (18), we obtain

$$
D=\frac{\rho_{l}}{2 \pi} d \phi d z \quad(\mathrm{C})
$$

for the electric flux density due to the line charge.

\section{Line Current}

If a current $I_{l}$ amperes flows along the $z$-axis, sheets of the $H$ 1-form will extend out radially from the current, as shown in Fig. 14. These are the surfaces of $d \phi$, so that by symmetry

$$
H=H_{0} d \phi
$$

where $H_{0}$ is a constant we need to find using Ampere's law. We choose the path $P$ in Ampere's law

$$
\oint_{P} H=\frac{d}{d t} \int_{A} D+\int_{A} J
$$

to be a loop around the $z$-axis. Assuming that $D=0$, the right-hand side of Ampere's law is equal to $I_{l}$. The left-hand side is the integral of $H$ over the loop

$$
\begin{aligned}
\oint_{P} H & =\int_{0}^{2 \pi} H_{0} d \phi \\
& =2 \pi H_{0} .
\end{aligned}
$$

The magnetic field intensity is then

$$
H=\frac{I_{l}}{2 \pi} d \phi(\mathrm{A})
$$

for the line current source.

\section{The EXterior Derivative AND MaXwell's LaWs IN POINT FORM}

In this section we introduce the exterior derivative and the generalized Stokes theorem and use these to express Maxwell's laws in point form. The exterior derivative is a single operator which has the gradient, curl, and divergence as special cases, depending on the degree of the differential form on which the exterior derivative acts. The exterior derivative has the symbol $d$, and can be written formally as

$$
d \equiv \frac{\partial}{\partial x} d x+\frac{\partial}{\partial y} d y+\frac{\partial}{\partial z} d z .
$$

The exterior derivative can be thought of as implicit differentiation with new differentials introduced from the left.

\section{A. Exterior Derivative of 0-Forms}

Consider the 0 -form $f(x, y, z)$. If we implicitly differentiate $f$ with respect to each of the coordinates, we obtain

$$
d f=\frac{\partial f}{\partial x} d x+\frac{\partial f}{\partial y} d y+\frac{\partial f}{\partial z} d z
$$

which is a 1-form, the exterior derivative of $f$. Note that the differentials $d x, d y$, and $d z$ are the exterior derivatives of the coordinate functions $x, y$, and $z$. The 1 -form $d f$ is dual to the gradient of $f$.

If $\phi$ represents a scalar electric potential, the negative of its exterior derivative is electric field intensity

$$
E=-d \phi .
$$

As noted earlier, the surfaces of the 1-form $E$ are equipotentials, or level sets of the function $\phi$, so that the exterior derivative of a 0 -form has a simple graphical interpretation.

\section{B. Exterior Derivative of 1-Forms}

The exterior derivative of a 1-form is analogous to the vector curl operation. If $E$ is an arbitrary 1-form $E_{1} d x+E_{2} d y+$ $E_{3} d z$, then the exterior derivative of $E$ is

$$
\begin{aligned}
d E= & \left(\frac{\partial}{\partial x} E_{1} d x+\frac{\partial}{\partial y} E_{1} d y+\frac{\partial}{\partial z} E_{1} d z\right) d x \\
& +\left(\frac{\partial}{\partial x} E_{2} d x+\frac{\partial}{\partial y} E_{2} d y+\frac{\partial}{\partial z} E_{2} d z\right) d y \\
& +\left(\frac{\partial}{\partial x} E_{3} d x+\frac{\partial}{\partial y} E_{3} d y+\frac{\partial}{\partial z} E_{3} d z\right) d z .
\end{aligned}
$$

Using the antisymmetry of the exterior product, this becomes

$$
\begin{aligned}
d E= & \left(\frac{\partial E_{3}}{\partial y}-\frac{\partial E_{2}}{\partial z}\right) d y d z \\
& +\left(\frac{\partial E_{1}}{\partial z}-\frac{\partial E_{3}}{\partial x}\right) d z d x \\
& +\left(\frac{\partial E_{2}}{\partial x}-\frac{\partial E_{1}}{\partial y}\right) d x d y
\end{aligned}
$$

which is a 2 -form dual to the curl of the vector field $E_{1} \hat{x}+$ $E_{2} \hat{\boldsymbol{y}}+E_{3} \hat{\boldsymbol{z}}$.

Any 1-form $E$ for which $d E=0$ is called closed and represents a conservative field. Surfaces representing different potential values can never meet. If $d E \neq 0$, the field is nonconservative, and surfaces meet or end wherever the exterior derivative is nonzero. 


\section{Exterior Derivative of 2-Forms}

The exterior derivative of a 2 -form is computed by the same rule as for 0 -forms and 1-forms: take partial derivatives by each coordinate variable and add the corresponding differential on the left. For an arbitrary 2-form $B$

$$
\begin{aligned}
d B= & d\left(B_{1} d y d z+B_{2} d z d x+B_{3} d x d y\right) \\
= & \left(\frac{\partial}{\partial x} B_{1} d x+\frac{\partial}{\partial y} B_{1} d y+\frac{\partial}{\partial z} B_{1} d z\right) d y d z \\
& +\left(\frac{\partial}{\partial x} B_{2} d x+\frac{\partial}{\partial y} B_{2} d y+\frac{\partial}{\partial z} B_{2} d z\right) d z d x \\
& +\left(\frac{\partial}{\partial x} B_{3} d x+\frac{\partial}{\partial y} B_{3} d y+\frac{\partial}{\partial z} B_{3} d z\right) d x d y \\
= & \left(\frac{\partial B_{1}}{\partial x}+\frac{\partial B_{2}}{\partial y}+\frac{\partial B_{3}}{\partial z}\right) d x d y d z
\end{aligned}
$$

where six of the terms vanish due to repeated differentials. The coefficient of the resulting 3 -form is the divergence of the vector field dual to $B$.

\section{Properties of the Exterior Derivative}

Because the exterior derivative unifies the gradient, curl, and divergence operators, many common vector identities become special cases of simple properties of the exterior derivative. The equality of mixed partial derivatives leads to the identity

$$
d d=0
$$

so that the exterior derivative applied twice yields zero. This relationship is equivalent to the vector relationships $\nabla \times(\nabla f)=0$ and $\nabla \cdot(\nabla \times \boldsymbol{A})=0$. The exterior derivative also obeys the product rule

$$
d(\alpha \wedge \beta)=d \alpha \wedge \beta+(-1)^{p} \alpha \wedge d \beta
$$

where $p$ is the degree of $\alpha$. A special case of (26) is

$$
\nabla \cdot(\boldsymbol{A} \times \boldsymbol{B})=\boldsymbol{B} \cdot(\nabla \times \boldsymbol{A})-\boldsymbol{A} \cdot(\nabla \times \boldsymbol{B}) .
$$

These and other vector identities are often placed in reference tables; by contrast, (25) and (26) are easily remembered.

The exterior derivative in cylindrical coordinates is

$$
d=\frac{\partial}{\partial \rho} d \rho+\frac{\partial}{\partial \phi} d \phi+\frac{\partial}{\partial z} d z
$$

which is the same as for rectangular coordinates but with the coordinates $\rho, \phi, z$ in the place of $x, y, z$. Note that the exterior derivative does not require the factor of $\rho$ that is involved in converting forms to vectors and applying the star operator. In spherical coordinates

$$
d=\frac{\partial}{\partial r} d r+\frac{\partial}{\partial \theta} d \theta+\frac{\partial}{\partial \phi} d \phi
$$

where the factors $r$ and $r \sin \theta$ are not found in the exterior derivative operator. The exterior derivative is

$$
d=\frac{\partial}{\partial u} d u+\frac{\partial}{\partial v} d v+\frac{\partial}{\partial w} d w
$$

in general orthogonal coordinates. The exterior derivative is much easier to apply in curvilinear coordinates than the vector derivatives; there is no need for reference tables of derivative formulas in various coordinate systems.

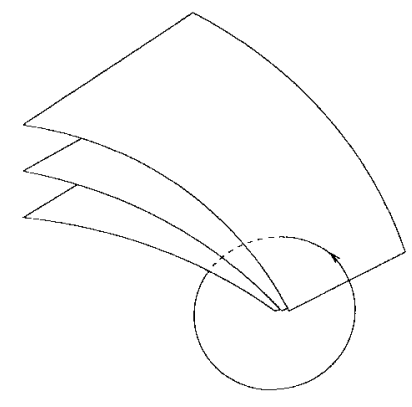

(a)

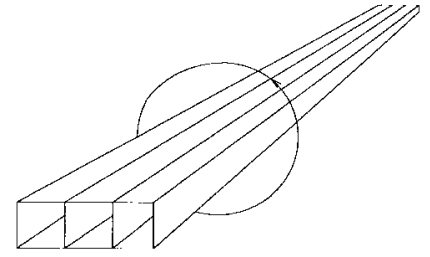

(b)
Fig. 15. The Stokes theorem for $\omega$ a 1-form. (a) The loop $b d M$ pierces three of the surfaces of $\omega$. (b) Three tubes of $d \omega$ pass through any surface $M$ bounded by the loop $b d M$.

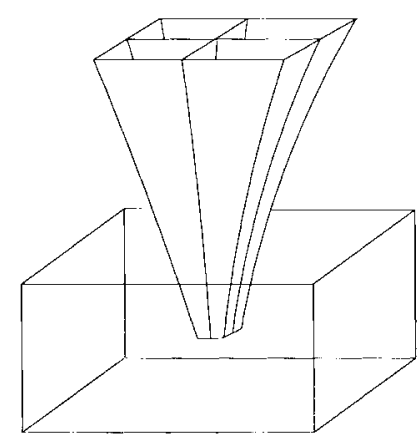

(a)

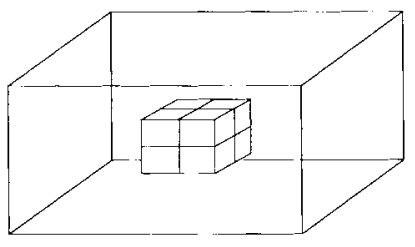

(b)
Fig. 16. Stokes theorem for $\omega$ a 2 -form. (a) Four tubes of the 2-form $\omega$ pass through a surface. (b) The same number of boxes of the 3 -form $d \omega$ lie inside the surface.

\section{E. The Generalized Stokes Theorem}

The exterior derivative satisfies the generalized Stokes theorem, which states that for any $p$-form $\omega$

$$
\int_{M} d \omega=\oint_{b d M} \omega
$$

where $M$ is a $(p+1)$-dimensional region of space and $b d M$ is its boundary. If $\omega$ is a 0 -form, then the Stokes theorem becomes

$$
\int_{a}^{b} d f=f(b)-f(a)
$$

This is the fundamental theorem of calculus.

If $\omega$ is a 1 -form, then $b d M$ is a closed loop and $M$ is a surface that has the path as its boundary. This case is analogous to the vector Stokes theorem. Graphically, the number of surfaces of $\omega$ pierced by the loop equals the number of tubes of the 2-form $d \omega$ that pass through the loop (Fig. 15).

If $\omega$ is a 2 -form, then $b d M$ is a closed surface and $M$ is the volume inside it. The Stokes theorem requires that the number of tubes of $\omega$ that cross the surface equal the number of boxes of $d \omega$ inside the surface, as shown in Fig. 16. This is equivalent to the vector divergence theorem. 
Compared to the usual formulations of these theorems,

$$
\begin{aligned}
f(b)-f(a) & =\int_{a}^{b} \frac{\partial f}{\partial x} d x \\
\oint_{b d A} \boldsymbol{E} \cdot d \boldsymbol{l} & =\int_{A} \nabla \times \boldsymbol{E} \cdot d \boldsymbol{A} \\
\oint_{b d V} \boldsymbol{D} \cdot d \boldsymbol{S} & =\int_{V} \nabla \cdot \boldsymbol{D} d v
\end{aligned}
$$

the generalized Stokes theorem is simpler in form and hence easier to remember. It also makes clear that the vector Stokes theorem and the divergence theorem are higher dimensional statements of the fundamental theorem of calculus.

\section{F. Faraday's and Ampere's Laws in Point Form}

Faraday's law in integral form is

$$
\oint_{P} E=-\frac{d}{d t} \int_{A} B .
$$

Using the Stokes theorem, taking $M$ to be the surface $A$, we can relate the path integral of $E$ to the surface integral of the exterior derivative of $E$

$$
\oint_{P} E=\int_{A} d E .
$$

By Faraday's law

$$
\int_{A} d E=-\frac{d}{d t} \int_{A} B .
$$

For sufficiently regular forms $E$ and $B$, we have that

$$
d E=-\frac{\partial}{\partial t} B
$$

since (33) is valid for all surfaces $A$. This is Faraday's law in point form. This law states that new surfaces of $E$ are produced by tubes of time-varying magnetic flux.

Using the same argument, Ampere's law becomes

$$
d H=\frac{\partial}{\partial t} D+J
$$

Ampere's law shows that new surfaces of $H$ are produced by tubes of time-varying electric flux or electric current.

\section{G. Gauss's Laws in Point Form}

Gauss's law for the electric flux density is

$$
\oint_{S} D=\int_{V} \rho .
$$

The Stokes theorem with $M$ as the volume $V$ and $b d M$ as the surface $S$ shows that

$$
\oint_{S} D=\int_{V} d D .
$$

Using Gauss's law in integral form (36)

$$
\int_{V} d D=\int_{V} \rho .
$$

We can then write

$$
d D=\rho
$$

This is Gauss's law for the electric field in point form. Graphically, this law shows that tubes of electric flux density can end only on electric charges. Similarly, Gauss's law for the magnetic field is

$$
d B=0 .
$$

This law requires that tubes of magnetic flux density never end; they must form closed loops or extend to infinity.

\section{H. Poynting's Theorem}

Using Maxwell's laws, we can derive a conservation law for electromagnetic energy. The exterior derivative of $S$ is

$$
\begin{aligned}
d S & =d(E \wedge H) \\
& =(d E) \wedge H-E \wedge(d H) .
\end{aligned}
$$

Using Ampere's and Faraday's laws, this can be written

$$
d S=-\frac{\partial}{\partial t} B \wedge H-E \wedge \frac{\partial}{\partial t} D-E \wedge J .
$$

Finally, using the definition (6) of $w$, this becomes

$$
d S=-\frac{\partial w}{\partial t}-E \wedge J
$$

At a point where no sources exist, a change in stored electromagnetic energy must be accompanied by tubes of $S$ that represent flow of energy toward or away from the point.

\section{Integrating Forms by Pullback}

We have seen in previous sections that differential forms give integration a clear graphical interpretation. The use of differential forms also results in several simplifications of the integration process itself. Integrals of vector fields require a metric; integrals of differential forms do not. The method of pullback replaces the computation of differential length and surface elements that is required before a vector field can be integrated.

Consider the path integral

$$
\int_{P} \boldsymbol{E} \cdot d \boldsymbol{l}
$$

The dot product of $\boldsymbol{E}$ with $d l$ produces a 1 -form with a single differential in the parameter of the path $P$, allowing the integral to be evaluated. The integral of the 1-form $E$ dual to $\boldsymbol{E}$ over the same path is computed by the method of pullback, as change of variables for differential forms is commonly termed. Let the path $P$ be parameterized by

$$
x=p_{1}(t), y=p_{2}(t), z=p_{3}(t)
$$

for $a<t<b$. The pullback of $E$ to the path $P$ is denoted $P^{*} E$, and is defined to be

$$
\begin{aligned}
P^{*} E= & P^{*}\left(E_{1} d x+E_{2} d y+E_{3} d z\right) \\
= & E_{1}\left(p_{1}, p_{2}, p_{3}\right) d p_{1}+E_{2}\left(p_{1}, p_{2}, p_{3}\right) d p_{2} \\
& +E_{3}\left(p_{1}, p_{2}, p_{3}\right) d p_{3} . \\
= & \left(E_{1}\left(p_{1}, p_{2}, p_{3}\right) \frac{\partial p_{1}}{\partial t}+E_{2}\left(p_{1}, p_{2}, p_{3}\right) \frac{\partial p_{2}}{\partial t}\right. \\
& \left.+E_{3}\left(p_{1}, p_{2}, p_{3}\right) \frac{\partial p_{3}}{\partial t}\right) d t
\end{aligned}
$$


Using the pullback of $E$, we convert the integral over $P$ to an integral in $t$ over the interval $[a, b]$

$$
\int_{P} E=\int_{a}^{b} P^{*} E
$$

Components of the Jacobian matrix of the coordinate transform from the original coordinate system to the parameterization of the region of integration enter naturally when the exterior derivatives are performed. Pullback works similarly for 2forms and 3-forms, allowing evaluation of surface and volume integrals by the same method. The following example illustrates the use of pullback.

Example 3 (Work Required to Move a Charge through an Electric Field): Let the electric field intensity be given by $E=2 x y d x+x^{2} d y-d z$. A charge of $q=1 C$ is transported over the path $P$ given by $\left(x=t^{2}, y=t, z=\right.$ $1-t^{3}$ ) from $t=0$ to $t=1$. The work required is given by

$$
W=-q \int_{P} 2 x y d x+x^{2} d y-d z
$$

which by (44) is equal to

$$
=-q \int_{0}^{1} P^{*}\left(2 x y d x+x^{2} d y-d z\right)
$$

where $P^{*} E$ is the pullback of the field 1-form to the path $P$

$$
\begin{aligned}
P^{*} E & =2\left(t^{2}\right)(t) 2 t d t+\left(t^{2}\right)^{2} d t-\left(-3 t^{2}\right) d t \\
& =\left(5 t^{4}+3 t^{2}\right) d t .
\end{aligned}
$$

Integrating this new 1 -form in $t$ over $[0,1]$, we obtain

$$
W=-\int_{0}^{1}\left(5 t^{4}+3 t^{2}\right) d t=-2 J
$$

as the total work required to move the charge along $P$.

\section{J. Existence of Graphical Representations}

With the exterior derivative, a condition can be given for the existence of the graphical representations of Section II. These representations do not correspond to the usual "tangent space" picture of a vector field, but rather are analogous to the integral curves of a vector field. Obtaining the graphical representation of a differential form as a family of surfaces is the general nontrivial, and is essentially equivalent to Pfaff's problem. By the solution of Pfaff's problem, each differential form may be represented graphically in two dimensions as families of lines. In three dimensions, a 1-form $\omega$ can be represented as surfaces if the rotation $\omega \wedge d \omega$ is zero. If $\omega \wedge d \omega \neq 0$, then there exist local coordinates for which $\omega$ has the form $d u+v d w$, so that it is the sum of two 1-forms, both of which can be graphically represented as surfaces. An arbitrary, smooth 2 -form in $R^{3}$ can be written locally in the form $f d y \wedge d h$, so that the 2 -form consists of tubes of $d g \wedge d h$ scaled by $f$.

\section{K. Summary}

Throughout this section, we have noted various aspects of the calculus of differential forms that simplify manipulations and provide insight into the principles of electromagnetics. The exterior derivative behaves differently depending on the degree of the form it operates on, so that physical properties of a field are encoded in the type of form used to represent it, rather than in the type of operator used to take its derivative. The generalized Stokes theorem gives the vector Stokes theorem and the divergence theorem intuitive graphical interpretations that illuminate the relationship between the two theorems. While of lesser pedagogical importance, the algebraic and computational advantages of forms cited in this section also aid students by reducing the need for reference tables or memorization of identities.

\section{The Interior Product and Boundary Conditions}

Boundary conditions can be expressed using a combination of the exterior and interior products. The same operator is used to express boundary conditions for field intensities and flux densities, and in both cases the boundary conditions have simple graphical interpretations.

\section{A. The Interior Product}

The interior product has the symbol $\lrcorner$. Graphically, the interior product removes the surfaces of the first form from those of the second. The interior product $d x\lrcorner d y=0$, since there are no $d x$ surfaces to remove. The interior product of $d x$ with itself is one. The interior product of $d x$ and $d x d y$ is $d x\lrcorner d x d y=d y$. To compute the interior product $d y\lrcorner d x d y$, the differential $d y$ must be moved to the left of $d x d y$ before it can be removed, so that

$$
\begin{aligned}
d y \perp d x d y & =-d y \perp d y d x \\
& =-d x .
\end{aligned}
$$

The interior product of arbitrary 1-forms can be found by linearity from the relationships

$$
\begin{aligned}
& d x\lrcorner d x=1, d x\lrcorner d y=0, d x\lrcorner d z=0 \\
& d y\lrcorner d x=0, d y\lrcorner d y=1, d y\lrcorner d z=0 \\
& d z\lrcorner d x=0, d z\lrcorner d y=0, d z\lrcorner d z=1 .
\end{aligned}
$$

The interior product of a 1-form and a 2-form can be found using

$$
\begin{aligned}
& d x\lrcorner d y \wedge d z=0, d x\lrcorner d z \wedge d x=-d z, d x\lrcorner d x \wedge d y=d y \\
& d y\lrcorner d y \wedge d z=d z, d y\lrcorner d z \wedge d x=0, d y\lrcorner d x \wedge d y=-d x \\
& d z\lrcorner d y \wedge d z=-d y, d z\lrcorner d z \wedge d x=d x, d z\lrcorner d x \wedge d y=0 .
\end{aligned}
$$

The following examples illustrate the use of the interior product. 
Example 4 (The Interior Product of Two 1-Forms): The interior product of $a=3 x d x-y d z$ and $b=4 d y+5 d z$ is

$$
\begin{aligned}
a\lrcorner b & =(3 x d x-y d z)\lrcorner(4 d y+5 d z) \\
& =12 x d x\lrcorner d y+15 x d x\lrcorner d z-4 y d z\lrcorner d y-5 y d z\lrcorner d z \\
& =-5 y
\end{aligned}
$$

which is the dot product $\boldsymbol{a} \cdot \boldsymbol{b}$ of the vectors dual to the 1 -forms $a$ and $b$.

Example 5 (The Interior Product of a 1-Form and a 2-Form): The interior product of $a=3 x d x-y d z$ and $c=4 d z d x+5 d x d y$ is

$$
\begin{aligned}
a\lrcorner c= & (3 x d x-y d z)\lrcorner(4 d z d x+5 d x d y) \\
= & 12 x d x\lrcorner d z d x+15 x d x\lrcorner d x d y-4 y d z\lrcorner d z d x \\
& -5 y d z\lrcorner d x d y \\
= & -12 x d z+15 x d y-4 y d x
\end{aligned}
$$

which is the 1-form dual to $-\boldsymbol{a} \times \boldsymbol{c}$, where $\boldsymbol{a}$ and $\boldsymbol{c}$ are dual to $a$ and $c$.

The interior product can be related to the exterior product using the star operator. The interior product of arbitrary forms $a$ and $b$ is

$$
a\lrcorner b=\star(\star b \wedge a)
$$

which can be used to compute the interior product in curvilinear coordinate systems. (This formula shows the metric dependence of the interior product as we have defined it; the interior product is usually defined to be the contraction of a vector with a form, which is independent of any metric.) The interior and exterior products satisfy the identity

$$
\alpha=n \wedge(n\lrcorner \alpha)+n\lrcorner(n \wedge \alpha)
$$

where $\alpha$ is an arbitrary form.

The Lorentz force law can be expressed using the interior product. The force 1-form $F$ is

$$
F=q(E-\boldsymbol{v}\lrcorner B)
$$

where $\boldsymbol{v}$ is the velocity of a charge $q$, and the interior product can be computed by finding the 1 -form dual to $v$ and using the rules given above. $F$ is dual to the usual force vector $\boldsymbol{F}$. The force 1-form has units of energy, and does not have as clear a physical interpretation as the usual force vector. In this case we prefer to work with the vector dual to $F$, rather than $F$ itself. Force, like displacement and velocity, is naturally a vector quantity.

\section{B. Boundary Conditions}

A boundary can be specified as the set of points satisfying $f(x, y, z)=0$ for some suitable function $f$. The surface normal 1-form is defined to be the normalized exterior derivative of $f$

$$
n=\frac{d f}{\sqrt{(d f\lrcorner d f)}} .
$$

The surfaces of $n$ are parallel to the boundary. Using a subscript 1 to denote the region where $f>0$, and a subscript

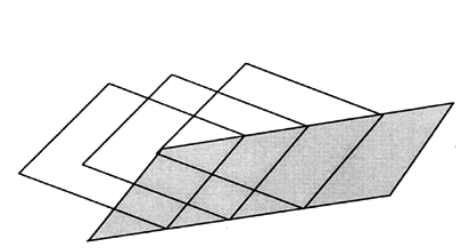

(a)

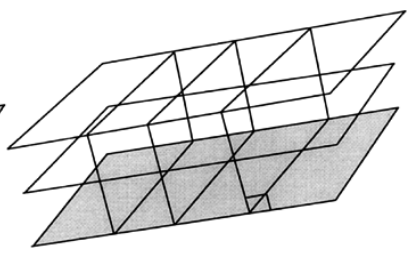

(b)

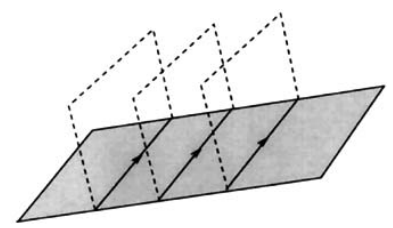

(c)

Fig. 17. (a) The 1 -form $H_{1}-H_{2}$. (b) The 2 -form $n \wedge\left(H_{1}-H_{2}\right)$. (c) The 1 -form $J_{s}$, represented by lines on the boundary. Current flows along the lines.

2 for $f<0$, the four electromagnetic boundary conditions can be written [18]

$$
\begin{aligned}
& n\lrcorner\left(n \wedge\left(E_{1}-E_{2}\right)\right)=0 \\
& n\lrcorner\left(n \wedge\left(H_{1}-H_{2}\right)\right)=J_{s} \\
& n\lrcorner\left(n \wedge\left(D_{1}-D_{2}\right)\right)=\rho_{s} \\
& n\lrcorner\left(n \wedge\left(B_{1}-B_{2}\right)\right)=0
\end{aligned}
$$

where $J_{s}$ is the surface current density 1 -form and $\rho_{s}$ is the surface charge density 2 -form. The operator $n\lrcorner n \wedge$ projects an arbitrary form to its component that has nonzero integral along the boundary.

\section{Surface Current}

The action of the operator $n\lrcorner n \wedge$ can be interpreted graphically, leading to a simple picture of the field intensity boundary conditions. Consider the field discontinuity $\mathrm{H}_{1}-\mathrm{H}_{2}$ shown in Fig. 17(a). The exterior product of $n$ and $H_{1}-H_{2}$ is a 2 -form with tubes that run parallel to the boundary, as shown in Fig. 17(b). The component of $H_{1}-H_{2}$ with surfaces parallel to the boundary is removed. The interior product $n\lrcorner\left(n \wedge\left(H_{1}-H_{2}\right)\right)$ removes the surfaces parallel to the boundary, leaving only surfaces perpendicular to the boundary, as in Fig. 17(c). Current flows along the lines where the surfaces intersect the boundary. The direction of flow along the lines of the 1-form can be found using the right-hand rule on the direction of $\mathrm{H}_{1}-\mathrm{H}_{2}$ in region 1 above the boundary.

The field intensity boundary conditions state that surfaces of the 1-form $\mathrm{H}_{1}-\mathrm{H}_{2}$ end along lines of the surface current density 1-form $J_{s}$. Surfaces of $E_{1}-E_{2}$ cannot intersect a boundary at all.

Unlike other electromagnetic quantities, $J_{s}$ is not dual to the vector $\boldsymbol{J}_{s}$. The direction of $\boldsymbol{J}_{s}$ is parallel to the lines of $J_{s}$ in the boundary, as shown in Fig. 17(c). ( $J_{s}$ is a twisted differential form, so that under coordinate inversion it transforms with a minus sign relative to a nontwisted 1-form. This property is discussed in detail in [3], [18], [21]. Operationally, the distinction can be ignored as long as one remains in right-handed coordinates.) $J_{s}$ is natural both mathematically and geometrically as a representation of 
surface current density. The expression for current through a path using the vector surface current density is

$$
I=\int_{\Gamma} \boldsymbol{J}_{s} \cdot(\hat{\boldsymbol{n}} \times d \hat{\boldsymbol{l}})
$$

where $\hat{\boldsymbol{n}}$ is a surface normal. This simplifies to

$$
I=\int_{P} J_{s}
$$

using the 1-form $J_{s}$. Note that $J_{s}$ changes sign depending on the labeling of regions one and two; this ambiguity is equivalent to the existence of two choices for $\hat{\boldsymbol{n}}$ in (52).

The following example illustrates the boundary condition on the magnetic field intensity.

Example 6 (Surface Current on a Sinusoidal Surface): A sinusoidal boundary given by $z-\cos y=0$ has magnetic field intensity $H_{1}=d x$ A above and zero below. The surface normal 1 -form is

$$
n=\frac{\sin y d y+d z}{\sqrt{1+\sin ^{2} y}} .
$$

By the boundary conditions given above

$$
\begin{aligned}
J_{s} & =n\lrcorner(n \wedge d x) \\
& \left.=\frac{1}{1+\sin ^{2} y}(\sin y d y+d z)\right\lrcorner(\sin y d y d z+d z d x) \\
& =\frac{d x+\sin ^{2} y d x}{1+\sin ^{2} y} \\
& =d x \text { (A). }
\end{aligned}
$$

The usual surface current density vector $\boldsymbol{J}_{\boldsymbol{s}}$ is $(\hat{\boldsymbol{y}}-$ $\sin y \hat{\boldsymbol{z}})\left(1+\sin ^{2} y\right)^{-1 / 2}$, which clearly is not dual to $d x$. The direction of the vector is parallel to the lines of $J_{s}$ on the boundary.

\section{Surface Charge}

The flux density boundary conditions can also be interpreted graphically. Fig. 18(a) shows the 2-form $D_{1}-D_{2}$. The exterior product $n \wedge\left(D_{1}-D_{2}\right)$ yields boxes that have sides parallel to the boundary, as shown in Fig. 18(b). The component of $D_{1}-D_{2}$ with tubes parallel to the boundary is removed by the exterior product. The interior product with $n$ removes the surfaces parallel to the boundary, leaving tubes perpendicular to the boundary. These tubes intersect the boundary to form boxes of charge (Fig. 18(c)). This is the 2 -form $\left.\rho_{s}=n\right\lrcorner(n \wedge$ $\left.\left(D_{1}-D_{2}\right)\right)$.

The flux density boundary conditions have as clear a graphical interpretation as those for field intensity: tubes of the difference $D_{1}-D_{2}$ in electric flux densities on either side of a boundary intersect the boundary to form boxes of surface charge density. Tubes of the discontinuity in magnetic flux density cannot intersect the boundary.

The sign of the charge on the boundary can be obtained from the direction of $D_{1}-D_{2}$ in region 1 above the boundary, which must point away from positive charge and toward negative charge. The integral of $\rho_{s}$ over a surface

$$
Q=\int_{S} \rho_{s}
$$

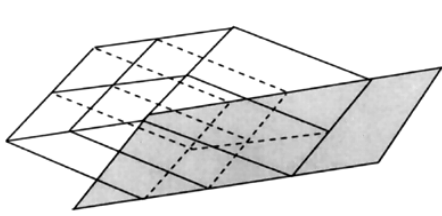

(a)

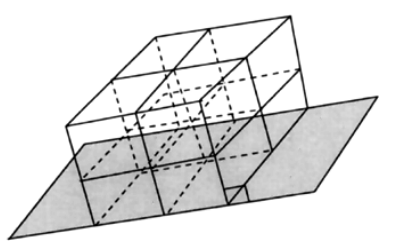

(b)

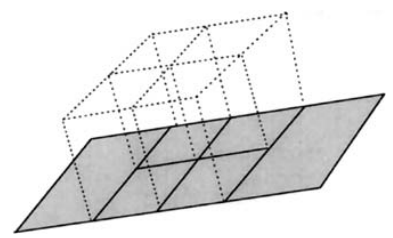

(c)

Fig. 18. (a) The 2 -form $D_{1}-D_{2}$. (b) The 3 -form $n \wedge\left(D_{1}-D_{2}\right)$, with sides perpendicular to the boundary. (c) The 2 -form $\rho_{s}$, represented by boxes on the boundary.

yields the total charge on the surface. Note that $\rho_{s}$ changes sign depending on the labeling of regions one and two. This ambiguity is equivalent to the existence of two choices for the area element $d A$ and orientation of the area $A$ in the integral $\int_{A} q_{s} d A$, where $q_{s}$ is the usual scalar surface charge density. Often, the sign of the value of the integral is known beforehand, and this subtlety goes unnoticed.

\section{CONCLUSION}

The primary pedagogical advantages of differential forms are the distinct representations of field intensity and flux density, intuitive graphical representations of each of Maxwell's laws, and a simple picture of electromagnetic boundary conditions. Differential forms provide visual models that can help students remember and apply the principles of electromagnetics. Computational simplifications also result from the use of forms: derivatives are easier to employ in curvilinear coordinates, integration becomes more straightforward, and families of related vector identities are replaced by algebraic rules. These advantages over traditional methods make the calculus of differential forms ideal as a language for teaching electromagnetic field theory.

The reader will note that we have omitted important aspects of forms. In particular, we have not discussed forms as linear operators on vectors, or covectors, focusing instead on the integral point of view. Other aspects of electromagnetics, including vector potentials, Green functions, and wave propagation also benefit from the use of differential forms.

Ideally, the electromagnetics curriculum set forth in this paper would be taught in conjunction with calculus courses employing differential forms. A unified curriculum, although desirable, is not necessary in order for students to profit from the use of differential forms. We have found that because of the simple correspondence between vectors and forms, the transition from vector analysis to differential forms is generally quite easy for students to make. Familiarity with vector analysis also helps students to recognize and appreciate 
the advantages of the calculus of differential forms over other methods.

We hope that this attempt at making differential forms accessible at the undergraduate level helps to fulfill the vision expressed by Deschamps [2] and others, that students obtain the power, insight, and clarity that differential forms offer to electromagnetic field theory and its applications.

\section{REFERENCES}

[1] C. Misner, K. Thorne, and J. A. Wheeler, Gravitation. San Francisco, CA: Freeman, 1973.

[2] G. A. Deschamps, "Electromagnetics and differential forms," Proc. IEEE, vol. 69, no. 6, pp. 676-696, June 1981.

[3] W. L. Burke, Applied Differential Geometry. Cambridge, U.K.: Cambridge Univ. Press, 1985.

[4] C. Nash and S. Sen, Topology and Geometry for Pphysicists. San Diego, CA: Academic Press, 1983.

[5] P. Bamberg and S. Sternberg, A Course in Mathematics for Students of Physics, vol. II. Cambridge, U.K.: Cambridge Univ. Press, 1988.

[6] H. Flanders, Differential Forms with Applications to the Physical Sciences. New York: Dover, 1963.

[7] Y. Choquet-Bruhat and C. DeWitt-Morette, Analysis, Manifolds and Physics. Amsterdam, The Netherlands: North-Holland, 1982.

[8] S. Hassani, Foundations of Mathematical Physics. Boston, MA: Allyn and Bacon, 1991

[9] R. Hermann, Topics in the Geometric Theory of Linear Systems. Brookline, MA: Math Sci. Press, 1984.

[10] D. Baldomir, "Differential forms and electromagnetism in 3-dimensional Euclidean space $R^{3}, " \quad$ Proc. Inst. Elec. Eng., vol. 133, no. 3, pp. 139-143, May 1986.

[11] N. Schleifer, "Differential forms as a basis for vector analysis-with applications to electrodynamics," Amer. J. Phys., vol. 51, no. 12, pp. 1139-1145, Dec. 1983.

[12] D. B. Nguyen, "Relativistic constitutive relations, differential forms, and the p-compound," Amer. J. Phys., vol. 60, no. 12, pp. 1137-1147, Dec. 1992.

[13] D. Baldomir and P. Hammond, "Global geometry of electromagnetic systems," Proc. Inst. Elec. Eng., vol. 140, no. 2, pp. 142-150, Mar. 1992.

[14] P. Hammond and D. Baldomir, "Dual energy methods in electromagnetics using tubes and slices," Proc. Inst. Elec. Eng., vol. 135, no. 3A, pp. $167-172$, Mar. 1988

[15] R. S. Ingarden and A. Jamiołkowski, Classical Electrodynamics,. Amsterdam, The Netherlands: Elsevier, 1985.
[16] S. Parrott, Relativistic Electrodynamics and Differential Geometry. New York: Springer-Verlag, 1987.

[17] W. Thirring, Classical Field Theory, vol. II. New York: SpringerVerlag, 1978

[18] K. F. Warnick, R. H. Selfridge, and D. V. Arnold, "Electromagnetic boundary conditions using differential forms," Proc. Inst. Elec. Eng., vol. 142 , no. 4, pp. 326-332, 1995.

[19] K. F. Warnick and D. V. Arnold, "Electromagnetic green functions using differential forms," J. Electromagn. Waves and Appl., vol. 10, no. 3, pp. 427-438, 1996

[20] _ _Differential forms in electromagnetic field theory," in Antennas and Propagation Symp. Proc., to be published, 1996.

[21] W. L. Burke, "Manifestly parity invariant electromagnetic theory and twisted tensors," J. Math. Phys., vol. 24, no. 1, pp. 65-69, Jan. 1983.

Karl F. Warnick is a National Science Foundation Graduate Fellow in Electrical Engineering at Brigham Young University, Provo, UT.

His current interests include nonlinear differential equations, differential geometry, topology, and electromagnetics in anisotropic media.

Richard H. Selfridge ( $\left.M^{\prime} 87\right)$ received the B.S. degree in physics from California State University, Sacramento, in 1987, and the M.S. and Ph.D. degrees in electrical engineering from the University of California, Davis, in 1980 and 1984, respectively.

From 1983 to 1987, he taught in the Department of Electrical and Computer Engineering at California State University, Sacramento. Since July of 1987, he has been a Professor of Electrical Engineering at Brigham Young University, Provo, UT. His research interests include modeling of conduction processes in semiconductors and mode locking of argon and dye lasers. Recently, his research has been directed toward the invention and fabrication of novel Dfiber devices. In addition, he is experimenting with approaches for teaching and using differential forms in electrical engineering.

Dr. Selfridge is a member of Sigma Xi and SPIE.

David V. Arnold received the B.S. and M.S. degrees from Brigham Young University, Provo, UT, and the Ph.D. degree from the Massachusetts Institute of Technology, Cambridge.

$\mathrm{He}$ is currently an Assistant Professor in the Electrical and Computer Engineering Department at Brigham Young University. His research interests are in electromagnetic theory and microwave remote sensing. 